\title{
Scaling analysis reveals the mechanism and rates of prion replication in vivo.
}

\author{
Georg Meis $1^{1, \dagger}$, Timothy Kurt ${ }^{2, \dagger}$, Itzel Condado-Morales ${ }^{1, \dagger}$, \\ Cyrus Bett ${ }^{2}$, Silvia Sorce ${ }^{3}$, Mario Nuvolone ${ }^{3,4}$, Thomas C. T. Michaels ${ }^{1}$, \\ Daniel Heinzer ${ }^{3}$, Merve Avar ${ }^{3}$, Samuel I. A. Cohen ${ }^{1,5}$, Simone Horneman ${ }^{3}$, \\ Adriano Aguzzi ${ }^{3}$, Christopher M. Dobson ${ }^{1}$, Christina J. Sigurdson ${ }^{2, *}$ \\ and Tuomas P. J. Knowles ${ }^{1,6, *}$ \\ $\nmid$ Contributed equally \\ ${ }^{1}$ Centre for Misfolding Diseases, Department of Chemistry, \\ University of Cambridge, Lensfield Road, Cambridge CB2 1EW, UK \\ ${ }^{2}$ Department of Pathology, UC San Diego, 9500 Gilman Drive, La Jolla, CA 92093, USA \\ ${ }^{3}$ Institute of Neuropathology, University Hospital of Zurich, 8091 Zurich, Switzerland \\ ${ }^{4}$ Amyloidosis Research and Treatment Center, Foundation IRCCS Policlinico San Matteo, \\ Department of Molecular Medicine, University of Pavia, 27100 Pavia, Italy \\ ${ }^{5}$ Wren Therapeutics, Clarendon House, Clarendon Road, Cambridge CB2 8FH, UK \\ ${ }^{6}$ Cavendish Laboratory, University of Cambridge, 19 JJ Thomson Avenue, \\ Cambridge CB3 OHE, UK
}

* To whom correspondence should be addressed;

E-mail: csigurdson@ucsd.edu, tpjk2@cam.ac.uk.

Prions consist of pathological assemblies of normal cellular prion protein and cause infectious neurodegenerative diseases, a phenomenon mirrored in many other prion-like neurodegenerative diseases. However, despite their key importance in disease, the individual processes governing this formation of pathogenic aggregates, as well as their rates, have remained challenging to elucidate in vivo. Here we bring together a mathematical framework with kinetics of the accumulation of prions in mice and microfluidic mea- 
surements of aggregate size to dissect the overall aggregation reaction into its constituent processes and quantify the reaction rates in mice. Taken together, the data show that multiplication of prions in vivo is slower than in in vitro experiments, but efficient when compared to other amyloid systems, and displays scaling behaviour characteristic of aggregate fragmentation. These results provide a framework for the determination of the mechanisms of disease-associated aggregation processes within living organisms.

Prions contain no conventional genetic information, yet, prions are infectious in a manner reminiscent of viruses $(1,2)$. To cause this behaviour, a small number of prions introduced upon infection have to be able to induce the formation of more prions, and this replication of infectious entities is thus the central process in prion diseases. This ability to replicate allows the disease to be initiated by a small number of particles, enabling prions to propagate from host to host and cause a rapidly progressive disorder. Although effects detrimental to the organism occur late in the development of the disease, and the detailed mechanism of toxicity is not yet fully established, the infection by and replication of prions is the crucial prerequisite for disease and thus understanding prion replication is central to understanding prion diseases (3-5). Prion disease is the archetypal aggregation-associated disease and in other neurodegenerative diseases the formed aggregates of proteins such as tau and $\alpha$-synuclein are often termed prion-like for similarities in their mechanism of propagation (6-8). Given this analogy with prion disease, the insights gained and framework developed here is likely to be of relevance to a wide range of disorders.

To avoid any confusion arising from the use of terminology from both the fields of prion diseases and of protein aggregation, we define here explicitly the terms used as: Infectious units are species capable of inducing the disease when introduced into a host (their relation to the species causing pathology is not required for this definition), infectivity is a measure of their concentration. In practice, infectivity is usually determined in dilution experiments, for 
example within mice (9) or in cell culture, through the Standard Scrapie Cell Assay (SSCA) (10). Aggregates are structures of several proteins in the $\operatorname{PrP}^{\mathrm{Sc}}$ state, the process that increases the size of a given aggregate is referred to as growth. Multiplication is the specific process that increases the number of aggregates, such as fragmentation of an existing aggregate. Replication is the overall process that converts molecules in the $\operatorname{PrP}^{\mathrm{C}}$ state to molecules in the $\mathrm{PrP}^{\mathrm{Sc}}$ state. Thus the rates of growth, multiplication and replication denote the rate of increase of size of a given aggregate, the rate at which a given aggregate produces new ones and the overall rate at which $\operatorname{PrP}^{\mathrm{C}}$ is converted to $\operatorname{PrP}^{\mathrm{Sc}}$.

It has been established that the conversion of monomeric cellular $\operatorname{PrP}^{\mathrm{C}}$ (here also referred to simply as monomer) to $\mathrm{PrP}^{\mathrm{Sc}}$ is at the core of the ability of prions to replicate (11). However, the molecular mechanisms, including the individual processes and their rates, by which existing infectious units can interact with $\mathrm{PrP}^{\mathrm{C}}$ and produce new infectious units have not been established in vivo. Numerous models have been proposed, including a direct conversion mechanism, whereby each protein in the $\mathrm{PrP}^{\mathrm{Sc}}$ state can catalyse the conversion of soluble $\operatorname{PrP}^{\mathrm{C}}$ molecules into $\mathrm{PrP}^{\mathrm{Sc}}$ via a hetero-dimer (12) and a fragmentation mechanism, whereby a large prion may break apart into two new prions (13) but it has remained challenging to verify either of these models in vivo. The majority of mechanistic studies have therefore to date focused instead on the kinetics of the aggregation of purified recombinant $\operatorname{PrP}$ in vitro $(11,14,15)$ and found the half time of aggregation to scale inversely with the square root of monomer concentration, which, as we and others have shown in the past (16), is consistent with a multiplication of aggregates by fragmentation. The relevance of these findings for the proliferation of prions in vivo remains, however, unclear, in particular as samples of aggregated, recombinant $\mathrm{PrP}^{\mathrm{Sc}}$ produced in vitro display considerably lower infectivity when injected into mice than the types of aggregates found in the brains of diseased organisms (17), suggesting a significant difference in structure, composition or mechanism. Overall, therefore, the mechanisms of prion replication 
on a molecular level remain to be established in vivo.

In order to address this challenge, we present a general framework for obtaining robust, representative parameters from in vivo data and relating them to fundamental mechanisms of multiplication and growth of protein aggregates. This approach is general enough to yield a continuous spectrum of behaviour, linking the previously proposed mechanisms $(13,18-24)$. Here, we show its power in analysing mechanisms in living systems by determining the rates for prion growth and multiplication in vivo.

\section{Results}

\section{Mechanistic analysis of prion multiplication.}

Chemical kinetics is the gold standard tool for discovering and verifying reaction mechanisms in molecular sciences, and this framework has recently been extended to protein aggregation where it fundamentally aids the mechanistic characterisation of complex reaction networks. In the present work, we apply this approach to aggregation taking place in a living system (Fig. 1a).

To obtain in vivo data amenable to mechanistic analysis within the framework of chemical kinetics, we studied the kinetics of $\mathrm{PrP}^{\mathrm{Sc}}$ formation in a total of 78 mice from four different lines; they include wild type (WT, also referred to as Prnp $^{+/+}$) mice and genetically modified mice that express approximately half (heterozygous WTx $P r n p^{0 / 0}$, also referred to as $P r n p^{0 /+}$ ), twice (heterozygous $\operatorname{tga} 20 \mathrm{x} \operatorname{Prnp}^{0 / 0}$, also referred to as $\operatorname{tga} 20 \mathrm{Prnp}^{0 /+}$ ) and three to four times (tga20, also referred to as $\operatorname{tg} \mathrm{a} 20 \mathrm{Prnp}^{+/+}$) the level of $\mathrm{PrP}^{\mathrm{C}}$ found in the WT mice. Crucially, studying the aggregation at different concentrations of $\operatorname{PrP}^{\mathrm{C}}$ allows the determination of the dependence of the aggregation rate on the $\operatorname{PrP}^{\mathrm{C}}$ concentration, which in turn can be linked to the nature of the replication mechanism. To initiate aggregation, the mice were inoculated intra-cerebrally with a well-characterized prion inoculum (RML5). We collected brain samples from each mouse line at multiple time points after inoculation until the onset of terminal disease, and measured levels 
of proteinase- $\mathrm{K}(\mathrm{PK})$ resistant and total $\mathrm{PrP}^{\mathrm{Sc}}$ (i.e. the sum of $\mathrm{PK}$ sensitive and $\mathrm{PK}$ resistant) as well as the levels of $\mathrm{PrP}^{\mathrm{C}}$ in the brains of inoculated mice. Additionally, we performed separate experiments to determine the time course of infectivity, using a standard scrapie cell assay, for a separate cohort of wild type mice (details see methods). To further increase robustness of our analysis, we combine our data with similar datasets from previous works. Finally, we used measurements of the molecular diffusivity of the aggregates to determined the average size of prions directly in brain homogenate.

\section{Infectivity and $\operatorname{PrP}^{\mathrm{Sc}}$ increase exponentially.}

An initially exponential increase in the number of aggregates is the hallmark of all aggregation mechanisms that include a multiplication and a growth process and emerges as a natural result of the auto-catalytic nature of self-replication, as we outline later and discuss in more detail in Supplementary Note 1 and Meisl et al. (25). Indeed, the results from our kinetic assay were consistent with an exponential increase in $\mathrm{PrP}^{\mathrm{Sc}}$ for the majority of the time-course, although $\mathrm{PrP}^{\mathrm{Sc}}$ amounts plateau late in the disease, as observed in previous studies $(26,27)$. To further verify the exponential increase, given that the initial measurements lie below the sensitivity of the ELISA, we also measured infectivity by SSCA as a function of time in wild type mice of a separate cohort to that used for the $\mathrm{PrP}^{\mathrm{Sc}}$ measurements (28). The infectivity is found it to increase exponentially, by several orders of magnitude, consistent with previously published data by Sandberg et al. (Fig. 1b) (27). Our interpretation of their data differ slightly from those presented by Sandberg et al. as we find that both the $\operatorname{PrP}^{\mathrm{Sc}}$ concentrations and the infectivity are consistent with an initially exponential increase and there is no evidence of one lagging behind the other (see Extended Data Fig. S1).

No $\mathrm{PrP}^{\mathrm{Sc}}$ was observed in age-matched uninfected controls, indicating that exposure to the $\mathrm{RML}$ isolate was essential for inducing $\mathrm{PrP}^{\mathrm{Sc}}$ formation. The average levels of $\operatorname{PrP}^{\mathrm{C}}$ were found 
to be slightly lower at the terminal disease stage with the most pronounced relative decrease (by 35\%) observed for WT (Prnp ${ }^{+/+}$) mice (Fig.2a). This finding is also in agreement with the observations of previous studies (26).
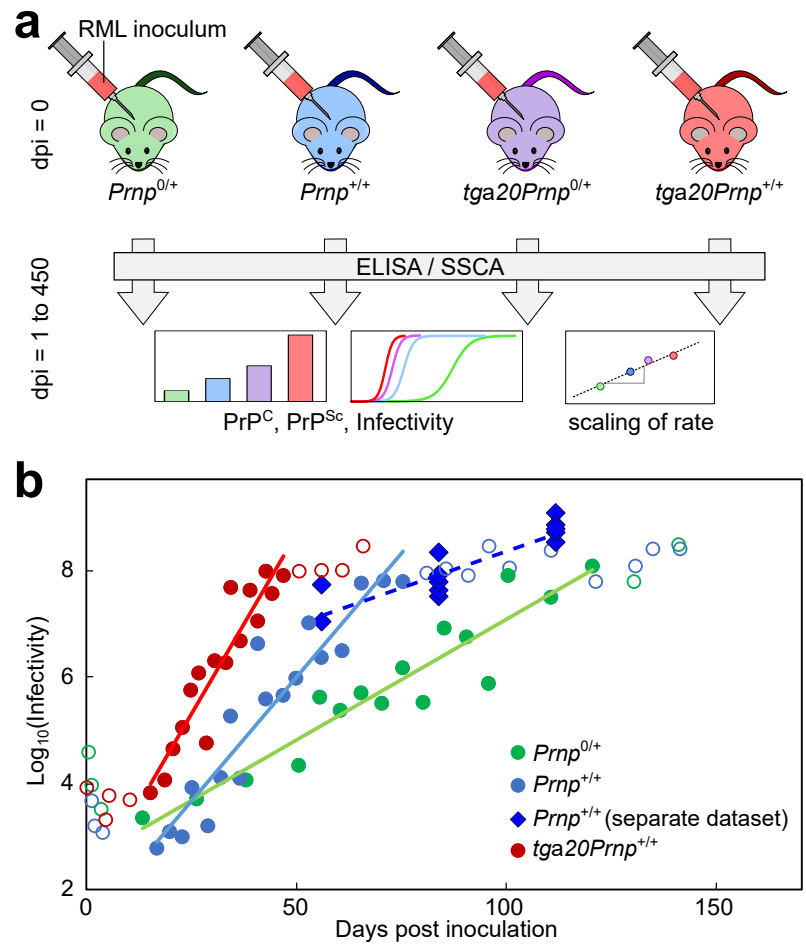

Figure 1: Principle of mechanistic analysis and increase of infectivity over time. (a) Mice of four different lines are inoculated with RML prions. At several time-points throughout disease, from inoculation up to 450 days post inoculation (dpi), the levels of $\mathrm{PrP}^{\mathrm{Sc}}$ and $\mathrm{PrP}^{\mathrm{C}}$ in mouse brains, as well as the infectivity of brain homogenate, is determined. The scaling of the rate of accumulation with $\mathrm{PrP}^{\mathrm{C}}$ amount serves as a guide to the mechanisms of multiplication. (b) Infectivity increases exponentially over the majority of the time-course, except the first few weeks after inoculation and close to terminal disease. The solid lines are straight line fits in logarithmic space to the filled circles, and show that the rate of increase is faster in mice with a higher $\operatorname{PrP}^{\mathrm{C}}$, the implications of which are discussed in the following sections. Open circles are data excluded from this fit. Circular points are data from Sandberg et al. (27), diamonds are data measured by SSCA by us in a cohort of wild type mice separate to that for the $\mathrm{PrP}^{\mathrm{Sc}}$ measurements $(28)$.

The reduction in $\mathrm{PrP}^{\mathrm{C}}$ concentration correlates with the appearance of pathological symptoms and may be the effect of higher order feedback processes, such as the organism's response 
to the accumulation of aggregates (26). In the context of an analysis of the kinetics of aggregate accumulation, the data provide the most robust constraint on the mechanistic details if the direct effect of the variation in a single parameter (in this case the $\operatorname{PrP}^{\mathrm{C}}$ concentration) can be measured. Given the complexity of any aggregate-induced response by the organism and the lack of knowledge of the exact nature and extent of the effects that a high concentration of aggregates has on the biochemical processes taking place in the organism, a kinetic analysis is thus most readily interpreted prior to the build-up of high levels of $\mathrm{PrP}^{\mathrm{Sc}}$ and the appearance of pathology.

We have therefore focused our analysis on the exponential stages of the $\mathrm{PrP}^{\mathrm{Sc}}$ accumulation, before its concentration plateaus and pathological symptoms of the mice become apparent. Infectivity increases by several orders of magnitude during this time period, making it the most important stage of the disease process to study in the context of prion replication. In practice, to show the robustness of our findings, we have employed 3 different methods to extract the rates of replication from these data: (1) a model free approach in which we determine the times at which a threshold concentration of $\operatorname{PrP}^{\mathrm{Sc}}$ is exceeded for each mouse line, (2) fits of a simple exponential to the pre-plateau phase, and (3) fits of a sigmoidal function that extends the initial exponential behaviour to produce a plateau at late times. The results of the last, most sophisticated method are shown here (Fig. 2). The other methods yield essentially unchanged results, which are shown in Extended Data Fig. S2 and Supplementary Note 2.

The function fitted in Fig. 2 is given by

$$
\left[\mathrm{PrP}^{\mathrm{Sc}}\right]=P_{\max }\left(\left(\frac{P_{\max }}{P_{0}}-1\right) e^{-\kappa t}+1\right)^{-1}
$$

where $\kappa$ is the exponential growth rate, $P_{0}$ the initial $\operatorname{PrP}^{\mathrm{Sc}}$ concentration and $P_{\max }$ the $\operatorname{PrP}^{\mathrm{Sc}}$ concentration at the plateau. This function approaches an exponential growth curve, $P_{0} e^{\kappa t}$, at early stages and allows for plateauing at late stages and in fact it emerges as the solution to the 

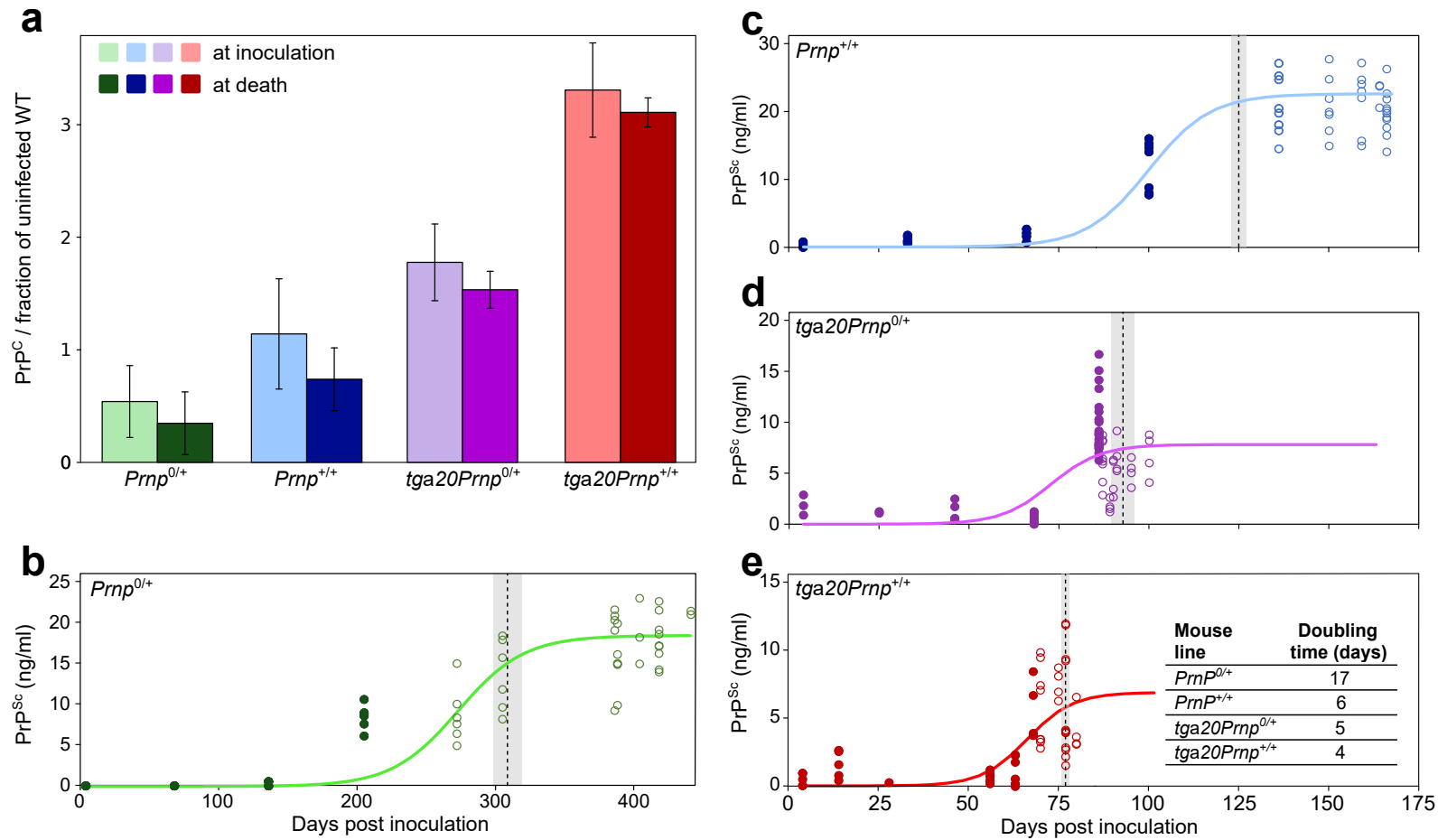

Figure 2: $\operatorname{PrP}^{\mathrm{C}}$ and $\operatorname{Pr} \mathrm{P}^{\mathrm{Sc}}$ concentrations over time for different mouse lines. (a) $\operatorname{PrP}^{\mathrm{C}}$ concentrations just after inoculation and shortly before terminal disease, for the each of the four different mouse lines. The concentrations are normalised to the $\mathrm{PrP}^{\mathrm{C}}$ concentration in an uninfected wild type mouse. (b-e) $\mathrm{PK}$ resistant $\mathrm{PrP}^{\mathrm{Sc}}$ concentration as a function of time, for each mouse line (the analogous plot for total $\mathrm{PrP}^{\mathrm{Sc}}$ concentration is given in the Extended Data Fig. S2). The filled circles are data-points in the pre-plateau region, the empty circles data-points in the plateau region. The solid lines are fits of equation (1) to all data-points. At each time-point samples from one or more mice were analysed, and 3-4 technical repeats of the ELISA assay of the same sample were performed (all technical repeats are shown). In total 78 mice were used (for detailed mouse numbers in each line see Extended Data S3). Dotted lines denote the approximate onset of symptoms (grey regions its standard deviation over remaining mice). Note the different time axis in $\mathbf{b}$; the other plots, $\mathbf{c}-\mathbf{e}$, share the same time axis.

logistic differential equation, the simplest description of auto-catalytic growth with a carrying capacity. Further details on its importance in describing aggregation reactions can be found in Meisl et al. (25). Setting $P_{0}$ and allowing $\kappa$ and $P_{\max }$ to vary reproduces the data well as shown in Fig. 2 (in Supplementary Note 2 we rationalise the choice of $P_{0}$ and show that the results are not sensitive to its specific value). The time to double the number of $\operatorname{PrP}^{\mathrm{Sc}}$ aggregates in 
the exponential phase, $t_{2}=\ln (2) / \kappa$, is approximately 4 days in the mice with the highest $\operatorname{PrP}^{\mathrm{C}}$ concentration (tga20) and 17 days in the mice with the lowest $\operatorname{PrP}^{\mathrm{C}}$ concentration $\left(\operatorname{Prnp} p^{0 /+}\right)$.

\section{Exponential rate depends on $\operatorname{PrP}^{\mathrm{C}}$ concentration.}

A key characteristic of each mechanism of protein aggregation is the dependence of $\kappa$ on the concentration of soluble precursor protein (29), which is quantified by the scaling exponent $\gamma$ as

$$
\kappa \propto\left[\mathrm{PrP}^{\mathrm{C}}\right]^{\gamma}
$$

The fact that only the relative variation in the exponential rates and monomer concentrations is required to determine this scaling gives it the necessary robustness to apply to in vivo experiments. Indeed, decades of work on the kinetic analysis of protein aggregation $(16,29,30)$ have shown that the two key characteristics of experimental data rich in mechanistic information are the nature of the time dependence (exponential or polynomial) and the concentration dependence given by $\gamma$. The level of infectivity of the inoculum or the absolute quantification of $\mathrm{PrP}^{\mathrm{Sc}}$ do not affect these quantities, making this approach uniquely suitable as a measure of the mechanism of aggregation in complex systems.

A graph of $\kappa$ versus the initial $\operatorname{PrP}^{\mathrm{C}}$ concentration on a double logarithmic plot allows the scaling exponent to be visualised (Eq. 2), see Fig. 3. Through this analysis of the rates of accumulation of $\mathrm{PK}$ resistant and total $\mathrm{PrP}^{\mathrm{Sc}}$ for the four mouse lines we find that the rates scale approximately with the square root of the $\operatorname{PrP}^{\mathrm{C}}$ concentration, i.e. $\gamma \approx 1 / 2$. As outlined above, we verified that our findings are not dependent on the specifics of the data analysis by extensively investigating the effect of changes in the fitting approaches (see Supplementary Note 2). 

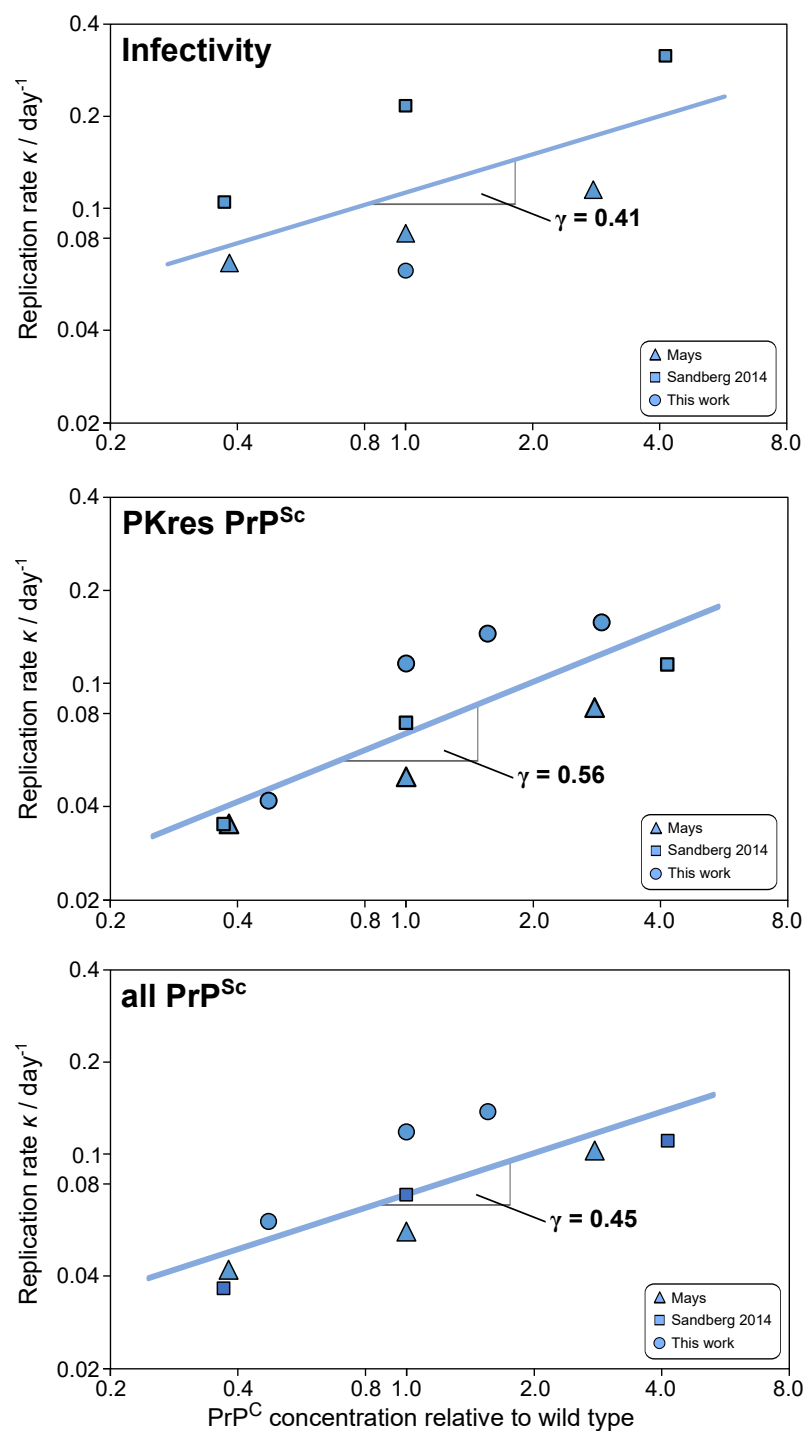

Figure 3: Scaling exponents of the rate of infectivity and of $\operatorname{PrP}^{\mathrm{Sc}}$ increase. Double logarithmic plots of the rate of accumulation, $\kappa$, of infectivity, $\mathrm{PK}$ resistant $\mathrm{PrP}^{\mathrm{Sc}}$ all $\mathrm{PrP}^{\mathrm{Sc}}$. Data included is from this work, Mays et al. (26) and Sandberg 2014 et al. (27). See Figs. 1b and 2, as well as Extended Data Fig. S2, S4 and S5 for primary data fits and Supplementary Note 3 for monomer concentrations and analysis details.

\section{Rates and $\operatorname{PrP}^{\mathrm{C}}$ dependence are consistent across datasets.}

Similar sets of data to those recorded here have been reported independently by Mays et al. (26) and by Sandberg et al. (27), for three mouse lines in each case. We analysed these data in the 
same manner as the data reported in the present study (see Supplementary Note 3 and Extended Data Figs. S4 and S5). Three quantities were measured in these studies: the concentration of $\mathrm{PK}$ resistant $\mathrm{PrP}^{\mathrm{Sc}}$, the concentration of total $\mathrm{PrP}^{\mathrm{Sc}}$ ( $\mathrm{PK}$ resistant and other species) and the infectivity. These studies all display an exponential increase in the measured quantity and determining the exponential rates to calculate the scaling for each of the quantities yields very similar results: The rate, $\kappa$, scales approximately as the square root of the $\operatorname{PrP}^{\mathrm{C}}$ concentration. Remarkably, this is true for both the $\operatorname{PrP}^{\mathrm{Sc}}$ concentration and the infectivity, although on average the rates of accumulation of infectivity are slightly higher than those of the accumulation of $\mathrm{PrP}^{\mathrm{Sc}}$ and the scaling is slightly lower. These differences may be a result of the differing sensitivities to small prion concentrations of the assays used to measure $\mathrm{PrP}^{\mathrm{Sc}}$ and infectivity.

The doubling times for $\mathrm{PK}$ resistant $\mathrm{PrP}^{\mathrm{Sc}}$ obtained in these other datasets are approximately 3 weeks and 1 week for the $\mathrm{Prnp}^{0 /+}$ and $\operatorname{tga} 20$, respectively, comparable to our data. We combine all data in Fig. 3 to obtain overall scaling exponents for the total and PK resistant PrPc concentrations, as well as the infectivity, which are all approximately $1 / 2$. Values of scaling exponents and errors for the different methods are summarised in Extended Data Fig. S6.

\section{Scaling exponent informs on replication mechanism.}

We now set out to link the experimental observations of the scaling behaviour to the underlying mechanism through a general framework for describing replication of aggregates in vivo. The general model is obtained by considering the fundamental classes of different processes that form the reaction network describing the conversion of monomeric proteins to aggregates. Two distinct types of processes are required to achieve replication of aggregated structures: (1) growth processes, which are responsible for the conversion of soluble $\operatorname{PrP}^{\mathrm{C}}$ into its aggregated form through addition to existing aggregates and (2) multiplication processes, which increase the number of aggregates. New aggregates in turn are able to grow through addition of solu- 
ble protein again, closing the positive feedback loop between growth and multiplication that is responsible for the overall exponential increase in aggregate mass (Fig. 4a). It is worth noting that the hetero-dimer mechanism whereby each molecule in the $\operatorname{PrP}^{\mathrm{Sc}}$ state can convert more $\operatorname{PrP}^{\mathrm{C}}(2)$, here referred to as direct monomer conversion, does not involve aggregation and hence does not distinguish between growth and multiplication processes. However, it can be obtained as a mathematical limit of the more general model used here and its predictions are shown to be inconsistent with the data (Fig. 4b).

Growth processes reflect the addition of soluble protein to existing $\mathrm{PrP}^{\mathrm{Sc}}$ aggregates so are easily described by one general mechanism. However, several different processes may be responsible for the formation of new aggregates. Under the conditions studied, the de-novo formation of aggregates from soluble $\mathrm{PrP}^{\mathrm{C}}$ alone, primary nucleation, was found to be negligible, as no $\mathrm{PrP}^{\mathrm{Sc}}$ accumulation was observed in mice inoculated with prion-free samples (this extremely slow rate of formation of aggregates directly from $\operatorname{PrP}^{\mathrm{C}}$ also explains the rarity of spontaneous prion disease). Thus, any production of new aggregates requires the presence of existing aggregates and is therefore a multiplication process. We can distinguish between two fundamental cases: the formation of new aggregates can depend on the concentration of aggregates alone, for example through the fragmentation of existing aggregates, or it can depend on both the concentration of existing aggregates and the concentration of monomers, for example in the case where the accessible surface of aggregates acts as a catalyst for the formation of new aggregates from soluble monomers in a secondary nucleation process (31-34). As the multiplication step is responsible for producing new aggregates, it also has to maintain the specific strain conformation. Differences of the kinetics between strains would, in this framework, be encapsulated in differing rates of growth and multiplication $(35,36)$. Finally, we also consider processes that result in the removal of aggregated species from the system $(37,38)$, for example autophagy or engulfment by microglia, or processes that prevent them from participating in the 
aggregation reaction, e.g. by incorporation into plaques, to complete the in vivo model. The detailed assumptions and limitations of this models are discussed in Supplementary Note 1. A more in depth treatment of the different classes of processes, explicitly considering variations of their rates with aggregate size, are considered in Meisl et al. (25). Briefly, the population of aggregates will generally consist of a range of species of different sizes, so to develop a general description one has to consider how the rate of both growth and multiplication depend on the size of the aggregate. This can be achieved by defining one continuous parameter each for growth and multiplication, which yields a continuous spectrum of behaviour that connects the limiting physical cases of self-replication and also applies to aggregates that are not linear in geometry. In essence, all mechanisms that include a multiplication step predict an exponential increase in aggregate mass $\left[\mathrm{PrP}^{\mathrm{Sc}}\right] \approx e^{\kappa t}$ where $\kappa$ is the replication rate. However, the dependence of this rate on the $\operatorname{PrP}^{\mathrm{C}}$ concentration can differ and is determined by the reaction orders with respect to $\mathrm{PrP}^{\mathrm{C}}$ of both the growth process and the multiplication process. More specifically, the replication rate is the geometric mean of the growth and the multiplication rates $\kappa=\sqrt{k_{\text {mult }} k_{\text {growth }}}$. This conclusion does not require the precise molecular mechanisms of growth and multiplication to be specified, but applies to all mechanisms that fall into the respective classes. In Fig. $4 \mathrm{~b}$ the scaling and the functional form of the increase of $\mathrm{PrP}^{\mathrm{Sc}}$ with time for a selection of common models are compared.

Using the data from the four independent experimental studies, we determined that the increase in $\mathrm{PrP}^{\mathrm{Sc}}$ concentration was exponential and that the scaling exponent was approximately 0.5 (see Fig. 3). Based on these observations several general classes of mechanisms can be discarded as inconsistent with the experimental data. The exponential increase excludes mechanisms that (i) lack multiplication and only involve the growth of inoculated (Fig. 4b II) or (ii) spontaneously formed aggregates (Fig. 4b III). Such behaviour is observed for example in the formation of actin filaments (39), but can be ruled out in these data of prions in mice. The 


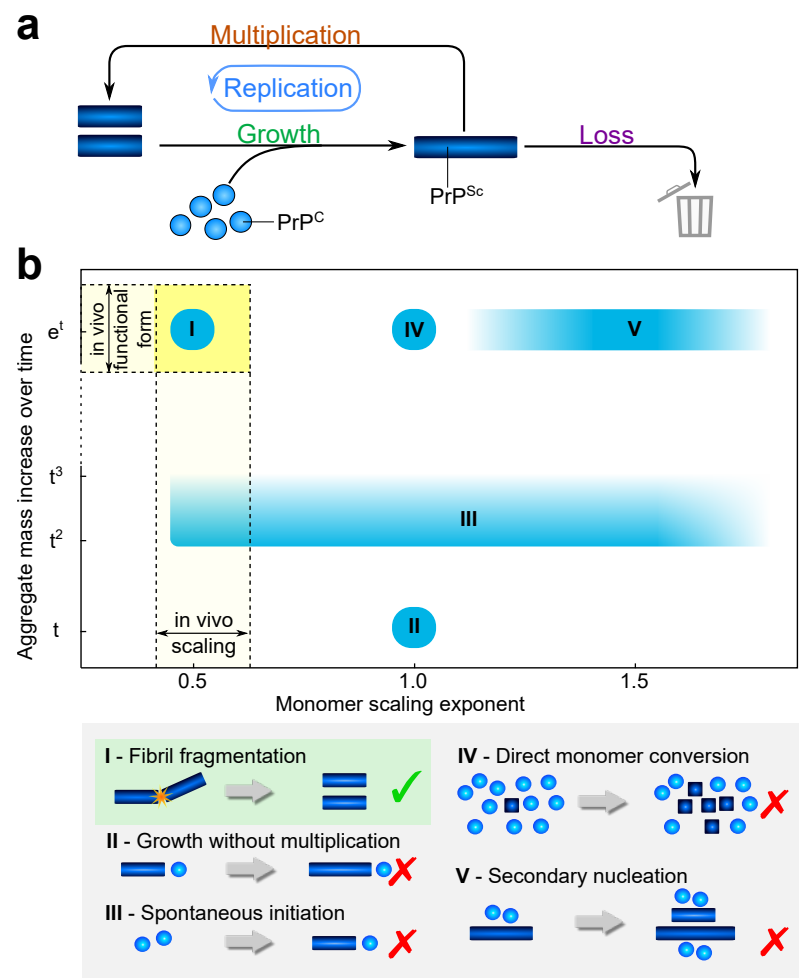

Figure 4: Reaction network and consistent mechanisms. (a) Monomeric $\operatorname{PrP}^{\mathrm{C}}$ is represented by blue spheres and $\mathrm{PrP}^{\mathrm{Sc}}$ aggregates are represented by blue bars. Aggregates can grow by incorporating more $\mathrm{PrP}^{\mathrm{C}}$, and can multiply for example by fragmentation. Growth and multiplication couple together in an auto-catalytic manner, the whole cycle is referred to as replication. Finally, the loss of aggregates by degradation and sequestration into plaques both have the effect of preventing the removed aggregates from further participating in the aggregation reaction (b) The scaling exponent, $\gamma$, is plotted against the functional form of the increase of aggregate mass with time, from linear to exponential. For the formation of linear aggregates without multiplication, the mass increase is linear in the absence of any spontaneous aggregate formation from monomer alone (II), or low-order polynomial if spontaneous formation of aggregates occurs (III). If aggregates are able to multiply, the mass increase is exponential (I, IV, V). Fragmentation of aggregates results in a scaling of approximately 0.5 (I), direct monomer conversion leads to a scaling of 1 (IV), and secondary nucleation gives a scaling 1 or above (V).

low value of the scaling exponent excludes the possibility of (iii) direct monomer conversion whereby each protein in the $\operatorname{PrP}^{\mathrm{Sc}}$ state may convert more $\operatorname{PrP}^{\mathrm{C}}$, also referred to as the heterodimer mechanism, which predicts a scaling of 1 (Fig. 4b IV), (iv) an aggregation mechanism that proceeds independently of the monomer concentration, which predicts a scaling of $0,(\mathrm{v})$ 
a mechanism of growth where oligomeric $\mathrm{PrP}^{\mathrm{C}}$ species, present at low concentrations and in equilibrium with monomer, are added to growing aggregates, which predicts a scaling of $>1$ (Fig. 4b V, scheme not shown) and (vi) a monomer concentration-dependent secondary nucleation process as is observed in vitro in the aggregation of the $\mathrm{A} \beta$ peptides associated with Alzheimer's disease (40), which also predicts a scaling of 1 or above (Fig. 4b V), depending on the number of monomeric species that take part in the nucleation reaction and the degree of saturation (41). Our findings are however in agreement with $\mathrm{PrP}^{\mathrm{Sc}}$ forming linear aggregates that multiply via fragmentation, $\gamma=0.5$ (Fig. $4 \mathrm{~b}$ I). They are also consistent with $\mathrm{PrP}^{\mathrm{Sc}}$ forming higher dimensional aggregates, such as bundles of aggregates that thicken as they grow, that are less likely to fragment the larger they become (25). It is interesting to compare this result with data obtained from in vitro studies $(16,42)$ which are indicative of fragmentation being the dominant mode of multiplication in the formation of linear fibrils of $\mathrm{PrP}^{\mathrm{Sc}}$. Therefore, although the formed structures and the rates of replication differ significantly, remarkably, the mechanism of replication in vivo is consistent with the mechanism in vitro.

\section{Prion size in brain homogenate by microfluidic sizing.}

A further experimentally accessible quantity, which allows deconvolution of the growth and multiplication rates from the overall effective rate of replication of $\mathrm{PrP}^{\mathrm{Sc}}$, is the average size of the aggregates formed. We used microfluidic diffusional sizing to determine the size of PK resistant $\mathrm{PrP}^{\mathrm{Sc}}$ aggregates in the brain homogenate of $\operatorname{tga} 20$ mice. A schematic of the platform is shown in Fig. 5. The technique is based on the fact that no turbulent mixing takes place under the conditions of laminar flow in the microfluidic device, and thus the movement of particles lateral to the flow direction is governed purely by diffusion and hence by their size. Having allowed the sample time diffuse, the concentrations are measured by antibody FRET using a POM antibody (43). Particle-based simulations of the diffusion of different sized species in the 
device (see Extended Data Fig. S7) allow us to relate the measured amounts of signal in each channel to the diffusion coefficient and thus the size of the detected particle (44). We found that the diffusion coefficient of PK resistant $\mathrm{PrP}^{\mathrm{Sc}}$ aggregates from the brains of prion-infected tga20 mice at terminal disease was over one order of magnitude larger than $\mathrm{PrP}^{\mathrm{C}}$ measured by the same method. To obtain the physical dimensions of the measured species, assumptions about their size have to be made, which is discussed in more detail below. However, the hydrodynamic radius related to a given diffusion coefficient is defined as the radius of a spherical particle that would diffuse at the same rate and can be calculated to assign a more easily interpretable quantity. It should not be confused with an actual size, if the particles are non-spherical.

\section{Comparison with other disease-associated proteins in vitro.}

To further probe the origin of the differences and similarities, and compare the observed data in mice to the aggregation of other disease-associated proteins in vitro, we set out to obtain values for the rates of the key processes of growth and multiplication. Growth and multiplication are connected in a positive feedback loop, Fig. 4a, and thus both contribute to overall prion replication, which is reflected in the fact that their rates only occur as a product in the rate of replication, $\kappa=\sqrt{k_{\text {mult }} k_{\text {growth }}}$. In order to dissect the contribution from the two processes, we require an additional measurement of an orthogonal property, such as the average number of PrP subunits in an aggregate. Such a measurement of the average aggregate size under in vivo conditions represents a major challenge. However, microfluidic diffusional sizing coupled to immunochemcial detection allows the determination of the size of $\mathrm{PK}$ resistant $\mathrm{PrP}^{\mathrm{Sc}}$ aggregates directly in brain homogenate from mice as detailed above (see Fig. 5). Given the measured hydrodynamic radius of $87 \pm 14 \mathrm{~nm}$ and accounting for the range of possible shapes of the aggregates and the possible presence of molecules other than $\mathrm{PrP}^{\mathrm{Sc}}$ in the aggregate, we obtain conservative bounds of between 100 and 100000 molecules of $\mathrm{PrP}^{\mathrm{C}}$ per aggregate on average 
a

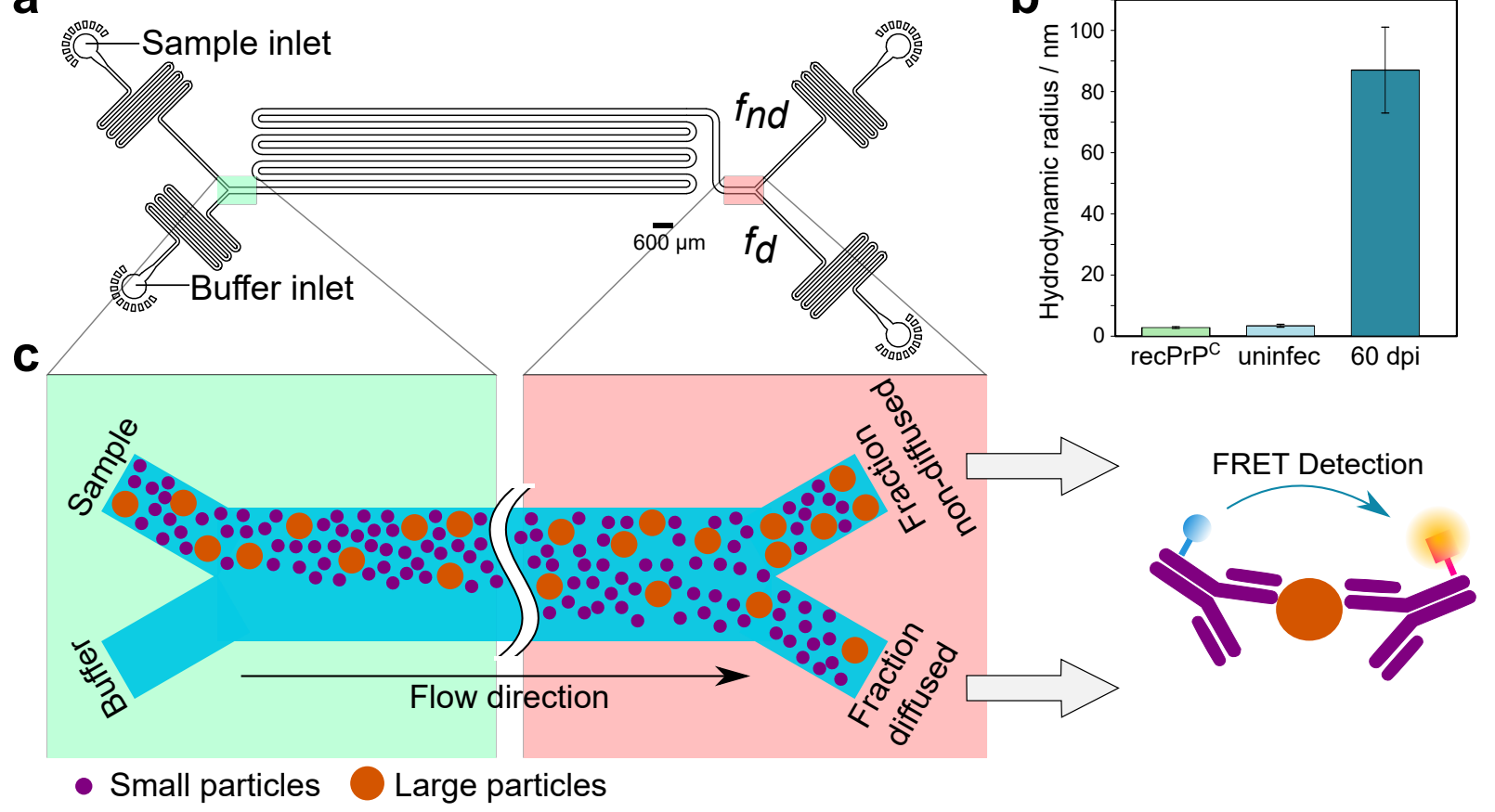

Figure 5: (a) Layout of the microfluidic device used to size prions in brain homogenate. The highlighted regions are shown schematically in c. (b) Measured hydrodynamic radii of recombinant PrP ("recPrP" ", $2.9 \pm 0.3 \mathrm{~nm}$ ), brain homogenate from uninfected tga20 mice ("uninfec", $3.5 \pm 0.5 \mathrm{~nm}$ ) and brain homogenate from tga20 mice 60 days post inoculation ("60 dpi", $87 \pm 14 \mathrm{~nm}$ ). Errors are the standard deviation from 3 technical repeats. Note that for prions, which are unlikely to be spherical, the hydrodynamic radius is likely an underestimation of their actual size. Conversion of hydrodynamic radii to number PrP subunits is discussed below. (c) Schematic showing how sample is injected along with buffer into the left side of the device. Particles diffuse laterally into the buffer stream, smaller particles diffuse further than larger ones. The microfluidic channel is eventually split into 2 parts, a diffused and an non-diffused fraction. The concentration of $\mathrm{PrP}^{\mathrm{Sc}}$ in each fraction is then determined using an antibody FRET assay.

(details see Supplementary Note 4). A number of previous studies have investigated the size of prions by a variety of methods, from electron microscopy imaging of brain homogenate with various levels of purification $(45,46)$ to live cell imaging $(47)$. While they suffer form different individual drawbacks, they generally find species hundreds of $\mathrm{nm}$ in length, with relatively low aspect ratios, consistent with aggregates composed of several hundreds or thousands of PrP molecules. A study by Silveira et al. (48) that measured the infectivity as a function of 
size, found that particles consisting only of tens of PrP molecules may already be infectious. However, even given the harsh treatment of their brain samples by sonication, the majority of species displayed a hydrodynamic radius between 30 and $60 \mathrm{~nm}$. Our results are therefore consistent with previous measurements and, as the method we present here is one of the mildest, are likely to be a good representation of the in vivo prion size.

The average number of subunits per aggregate, $\mu$, is related to the rates of multiplication, $k_{\text {mult }}$, and growth, $k_{\text {growth }}$, by $k_{\text {mult }}=\kappa / \mu$. This decomposition into growth and multiplication rates is valid for any general growth-multiplication type mechanism, as we outline in Supplementary Note 1 and Meisl et al. (25). For reference, we estimate the rate of multiplication in the brain of tga20 mice for an intermediate average prion size of 3000 monomers per aggregate to be $5 \cdot 10^{-10} \mathrm{~s}^{-1}$ and the rate of growth to be $5 \cdot 10^{-3} \mathrm{~s}^{-1}$, from the average of the rates from all $\operatorname{tga} 20$ datasets. To compare these results to the aggregation of other proteins which has been measured in vitro, we use previously published values for the rate constants and extrapolate these to obtain the rates of growth, multiplication and replication at a protein concentration of $135 \mathrm{nM}$ (see Supplementary Note 5), which corresponds to the concentration measured in tga20 mice (26), see Fig. 6. There are clear differences between prion replication in mice and the aggregation of purified PrP in vitro. Most notably, the multiplication rate of prions is orders of magnitude lower than that of purified PrP. These differences in rates quantify the effect of various cellular mechanisms, such as chaperones, whose function is to prevent protein aggregation. In light of this significant decrease of PrP replication in vivo compared to in vitro, it is quite remarkable that the rate of replication of prions is still significantly higher than the in vitro rates of both $\alpha$-synuclein and tau, proteins whose aggregation plays a central role in Parkinson's and Alzheimer's diseases. The high rate associated with prion replication may lie at the core of the reason why prion diseases are generally significantly more infectious and progress more rapidly than these other aggregation-related disorders. 

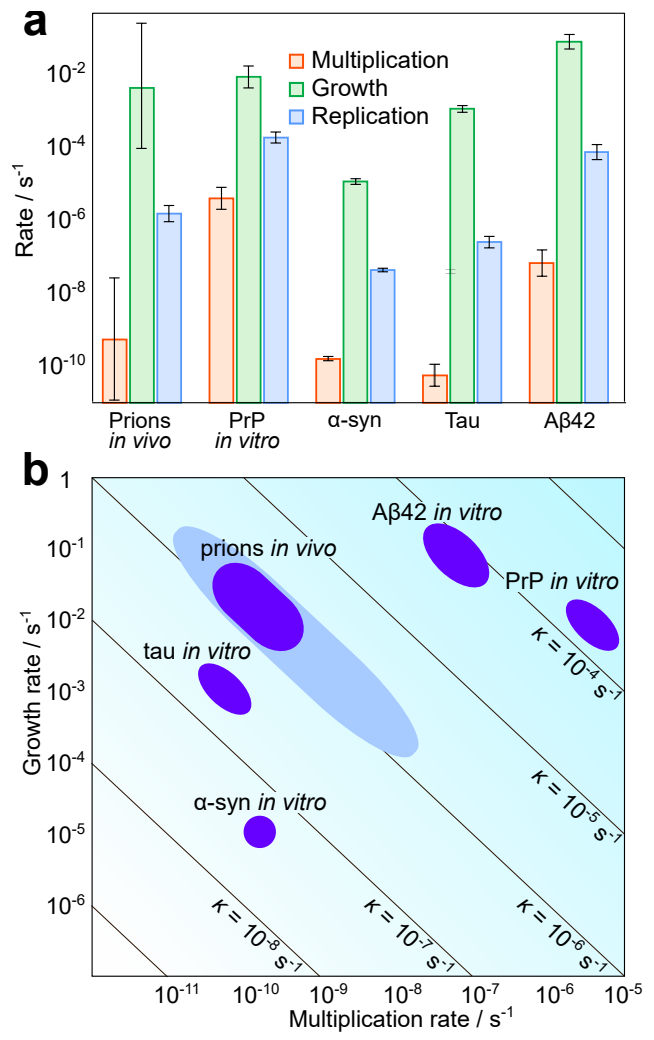

Figure 6: Individual rates in vivo and in vitro. (a) Rates of growth, multiplication and overall replication for prions in tga20 mice and a range of other in vitro systems. (b) The growth rate is plotted against the multiplication rate, lines of constant replication rate are shown as diagonals. For prions in vivo, the rates obtained assuming prions are elongated structures and consist between 3000 and 30000 PrP monomers are shown in dark blue, the bounds obtained without these assumptions are shown in light blue. The rate constants and error bars were obtained: for PrP and $\alpha$-synuclein from Sang et al. (42), for tau from Kundel et al. (49), and for A $\beta 42$ from Meisl et al. (50). The growth and replication rates for the in vitro systems were then calculated at a monomer concentration of $135 \mathrm{nM}$, which is the concentration of $\mathrm{PrP}^{\mathrm{C}}$ in $\operatorname{tga} 20$ mice. The error bars for the replication rate of prions are the ranges of rates obtained from the different analysis methods and different datasets, the error bars for multiplication and growth are derived from upper and lower bounds on the average aggregate size (see Supplementary Notes 4 and 5).

\section{Discussion}

We have established a general framework for describing aggregation in vivo by using a robust measure, the scaling exponent. The rates and scaling exponents obtained in this manner can 
be linked to the underlying molecular processes, thus giving insights into the mechanisms of self-replication. Rates of the processes in this mechanism can be estimated and our results reveal that the rate of multiplication of prions in vivo is slower than that of aggregates of pure $\mathrm{PrP}^{\mathrm{Sc}}$ in vitro, but faster than that of $\alpha$-synuclein and tau in vitro. We designed the application of this method for the case of mammalian prions, but the approach presented here is general and we envisage that it will be widely applicable in identifying the mechanism and rates of self-replication for a range of other aggregating systems in vivo.

\section{References}

1. A. Aguzzi, M. Polymenidou, Cell 116, 313 (2004).

2. S. Prusiner, Science 216, 136 (1982).

3. M. K. Sandberg, H. Al-Doujaily, B. Sharps, A. R. Clarke, J. Collinge, Nature 470, 540 (2011).

4. M. Eigen, Biophys Chem 63, A1 (1996).

5. F. Cohen, et al., Science 264, 530 (1994).

6. A. Mudher, et al., Acta Neuropathologica Communications 5, 99 (2017).

7. M. Goedert, F. Clavaguera, M. Tolnay, Trends in Neurosciences 33, 317 (2010).

8. G. Meisl, T. P. Knowles, D. Klenerman, Current Opinion in Neurobiology 61, 58 (2020). Neurobiology of Disease.

9. S. B. Prusiner, et al., Annals of Neurology 11, 353 (1982).

10. P.-C. Klohn, L. Stoltze, E. Flechsig, M. Enari, C. Weissmann, Proceedings of the National Academy of Sciences 100, 11666 (2003). 
11. B. Caughey, D. A. Kocisko, G. J. Raymond, P. T. Lansbury, Chemistry \& Biology 2, 807 (1995).

12. S. B. Prusiner, Science 252, 1515 (1991).

13. J. H. Come, P. E. Fraser, P. T. Lansbury, Proc Natl Acad Sci U S A 90, 5959 (1993).

14. K.-W. Leffers, et al., Biological Chemistry 386, 569 (2005).

15. J. Stöhr, et al., Proc Natl Acad Sci U S A 105, 2409 (2008).

16. T. P. J. Knowles, et al., Science 326, 1533 (2009).

17. A. Aguzzi, Nat Cell Biol 6, 290 (2004).

18. M. A. Nowak, D. C. Krakauer, A. Klug, R. M. May, Integrative Biology 1, 3 (1998).

19. J. Masel, V. A. Jansen, M. A. Nowak, Biophys Chem 77, 139 (1999).

20. T. R. Serio, et al., Science 289, 1317 (2000).

21. T. Poeschel, N. V. Brilliantov, C. Froemmel, Biophys J 85, 3460 (2003).

22. M. L. Greer, L. Pujo-Menjouet, G. F. Webb, J. Theor. Biol. 242 (2006).

23. V. Calvez, et al., Mathematical Biosciences 217, 88 (2009).

24. R. V. Kulkarni, A. Slepoy, R. R. P. ingh, D. L. Cox, F. Pázmándi, Biophys J 85, 707 (2003).

25. G. Meisl, A. J. Dear, T. C. T. Michaels, T. P. J. Knowles, arXiv 2008.09699 (2020).

26. C. E. Mays, et al., J Virol. 24, 12418 (2015).

27. M. K. Sandberg, et al., Nat Commun 5, 4347 (2014). 
28. S. Sorce, et al., PLOS Pathogens 16, 1 (2020).

29. G. Meisl, et al., Chem. Sci. 8, 7087 (2017).

30. G. Meisl, et al., Nature Protocols 11, 252 (2016).

31. F. A. Ferrone, J. Hofrichter, W. A. Eaton, J Mol Biol 183, 611 (1985).

32. R. Gaspar, et al., Quarterly Reviews of Biophysics 50 (2017).

33. M. Törnquist, et al., Chem. Commun. 54, 8667 (2018).

34. M. Törnquist, et al., Proceedings of the National Academy of Sciences 117, 11265 (2020).

35. A. Aguzzi, M. Heikenwalder, M. Polymenidou, Nature Reviews Molecular Cell Biology 8, 552 (2007).

36. A. Lau, et al., Nature Neuroscience 23, 21 (2020).

37. D. Peretz, et al., Nature 412, 739 (2001).

38. J. G. Safar, et al., Journal of General Virology 86, 2913 (2005).

39. F. Oosawa, Results Probl Cell Differ 32, 9 (2001).

40. S. I. A. Cohen, et al., Proceedings of the National Academy of Sciences 110, 9758 (2013).

41. A. J. Dear, et al., The Journal of Chemical Physics 152, 045101 (2020).

42. J. C. Sang, et al., Journal of the American Chemical Society 140, 14789 (2018).

43. B. A. Ballmer, et al., Journal of Biological Chemistry (2017).

44. T. Müller, et al., International Journal of Nonlinear Sciences and Numerical Simulation 17, 175 (2016). 
45. C. Terry, et al., Open Biology 6, 160035 (2016).

46. A. Wenborn, et al., Scientific Reports 5, 10062 (2015).

47. A. Rouvinski, et al., Journal of Cell Biology 204, 423 (2014).

48. J. R. Silveira, et al., Nature 237, 257 (2005).

49. F. Kundel, et al., ACS Chemical Neuroscience 9, 1276 (2018). PMID: 29590529.

50. G. Meisl, X. Yang, C. M. Dobson, S. Linse, T. P. J. Knowles, Chem. Sci. 8, 4352 (2017).

51. M. Fischer, et al., EMBO J 15, 1255 (1996).

52. H. Büeler, et al., Cell 73, 1339 (1993).

53. G. Kärber, Naunyn-Schmiedebergs Archiv für experimentelle Pathologie und Pharmakologie 162, 480 (1931).

54. A. L. Lau, et al., Proceedings of the National Academy of Sciences 104, 11551 (2007).

55. M. Polymenidou, et al., PLOS ONE 3, 1 (2008).

56. S. P. Mahal, C. A. Demczyk, E. W. Smith, P.-C. Klohn, C. Weissmann, Methods Mol Biol 459, 49 (2008).

57. M. Nuvolone, et al., Journal of Experimental Medicine 213, 313 (2016).

58. J. Brody, P. Yager, Sensors and Actuators A: Physical 58, 13 (1997).

59. P. Arosio, et al., ACS Nano 10, 333 (2016). PMID: 26678709. 


\section{Methods}

Mice used. For $\mathrm{PrP}^{\mathrm{Sc}}$ measurements: WT (C57BL/6), WT x Prnp ${ }^{0 / 0}$, tga20 and tga20 x $\operatorname{Prnp}^{0 / 0}$ transgenic mice. Both male and female mice were utilized in this study. The mice were inoculated at approximately 2 months of age (62 days \pm 3 days). For SSCA measurements and size determination: six-week old C57BL/6J male mice purchased from Charles River (Germany) were inoculated. Mice were maintained on a $12 \mathrm{~h}: 12 \mathrm{~h}$ light/dark cycle at an ambient temperature $\left(21-23^{\circ} \mathrm{C}\right)$ and $50-60 \%$ humidity.

\section{Inoculation of mice for $\operatorname{PrP}^{\mathrm{Sc}}$ and $\operatorname{PrP}^{\mathrm{C}}$ measurements WT (C57BL/6), WT x Prnp ${ }^{0 / 0}$,} $\operatorname{tga} 20$ or $\operatorname{tga} 20 \times$ Prnp $^{0 / 0}$ transgenic mice (groups of $\mathrm{n}=10-12$ mice) of either sex were intracerebrally inoculated into the left parietal cortex with $30 \mu \mathrm{l}$ of $0.01 \%$ brain homogenate containing RML5 (passage 5 of Rocky Mountain Laboratory strain mouse scrapie prions). The titer of the RML5 inoculum was $8.9 \log \left(\mathrm{LD}_{50}\right) \mathrm{g}^{-1}$ of brain tissue. $\operatorname{tga} 20$ and $P r n p^{0 / 0}$ mice have been previously described $(51,52)$, and were maintained under specific pathogen-free conditions. Mice were monitored three times weekly, and prion disease was diagnosed according to clinical criteria including ataxia, kyphosis, stiff tail, hind leg clasp, and hind leg paresis. The mice were sacrificed at time-points throughout the disease incubation period or at the onset of terminal disease when showing signs including weight loss, tremors, slow movements, and kyphosis.

Inoculation of mice for infectivity measurements C57BL/6J male mice were purchased from Charles River (Germany) and allowed at least one week of habituation before inoculations. Experimental manipulations were performed in compliance with the Swiss Animal Protection Law, and approved by the Veterinary office of the Canton Zurich (animal permits 41/2012, 90/2013, ZH040-15). Six-week old C57BL/6J male mice were injected under isoflurane anesthesia in the right hemisphere with $30 \mu$ of RML6 (passage 6 of Rocky Mountain 
Laboratory strain mouse-adapted scrapie prions, available upon request) at a $10^{-2}$ dilution of a $10 \%$ homogenate $\left(10 \% \mathrm{w} / \mathrm{v}\right.$ in $0.32 \mathrm{M}$ Sucrose, $10^{9.02} \mathrm{LD}_{50}$ units per $\left.\mathrm{mL}\right)(53)$.

At selected time points after prion inoculation, mice were deeply anesthetized and transcardially perfused with cold PBS before brain collection. Animal experiments were performed in compliance with the Swiss Animal Protection Law, under the approval of the Veterinary office of the Canton Zurich (animal permits 41/2012, 90/2013, ZH040-15). Intracerebral injections were performed under isoflurane anesthesia. All efforts were made to prevent or minimize animal discomfort and suffering.

Sample preparation for $\operatorname{PrP}^{\mathrm{C}}$ measurements $10 \%$ brain homogenates from RML-infected mice shortly after inoculation and at the terminal stage were measured by the BCA assay. $200 \mu \mathrm{g}$ of each brain sample was diluted to $60 \mu \mathrm{l}$ total volume using phosphate buffered saline (PBS). Samples were digested using Benzonase ${ }^{\mathrm{TM}}$ (Millipore) with gentle shaking for $20 \mathrm{~min}$ at $37^{\circ} \mathrm{C}$, then mixed with $20 \mu \mathrm{l} 4 \%$ sarkosyl with shaking for $20 \mathrm{~min}$ at $37^{\circ} \mathrm{C}$. Samples were then centrifuged at $16,000 \mathrm{xg}$ for $30 \mathrm{~min}$ at $4^{\circ} \mathrm{C}$. The supernatants were carefully collected and stored at $-80^{\circ} \mathrm{C} .50 \mu \mathrm{l}$ of each sample was injected onto a Fast Performance Liquid Chromatography (FPLC) column (Superose 6, 3.2/30) using running buffer (50 mM NH $\mathrm{m}_{4} \mathrm{OAc}, 0.1 \%$ sarkosyl, $\mathrm{pH} 8.5$ ) at a flow rate of $60 \mu \mathrm{l} / \mathrm{min}$ to collect $24 \times 60 \mu \mathrm{l}$ fractions (see Fig. S8A-C). Fractions 16-19 were pooled and $50 \mu \mathrm{l} /$ well aliquots were loaded into the ELISA plate (Extended Data Fig. S8). Samples from tga20 mice were diluted 1:2 due to the higher $\operatorname{PrP}^{\mathrm{C}}$ levels of this mouse line, and ELISA measurements from these samples were corrected for this dilution factor.

Measurement of PK-resistant PrP $\mathbf{P}^{\mathrm{Sc}}$ Protein concentrations in $10 \%$ brain homogenates in PBS were measured by bicinchoninic assay (BCA) and samples were normalized using PBS. $27 \mu \mathrm{l}$ aliquots of each sample were mixed with $1.5 \mu \mathrm{l} 20 \%$ sarcosyl at $37^{\circ} \mathrm{C}$ for $15 \mathrm{~min}$ before addition of $1.5 \mu \mathrm{l} \mathrm{PK}$ (Roche) to a final concentration of $100 \mu \mathrm{g} / \mathrm{mL}$ and incubation at $37^{\circ} \mathrm{C}$ 
for $30 \mathrm{~min}$. To facilitate PK digestion samples from tga20 mice only were brought to a final concentration of $0.375 \%$ sodium dodecyl sulphate (SDS) and were digested at $37^{\circ} \mathrm{C}$ for 30 min followed by $10 \mathrm{~min}$ at $45^{\circ} \mathrm{C}$. Protease-digestion was stopped by addition of $1.5 \mu 1100 \mathrm{mM}$ phenylmethanesulfonyl fluoride (PMSF) and mixed with $11 \mu \mathrm{l} 8 \mathrm{M} \mathrm{Gdn}-\mathrm{HCl}$ prior to incubation for $5 \mathrm{~min}$ at $80^{\circ} \mathrm{C}$. Samples were then diluted by addition of $362 \mu \mathrm{l}$ tris buffered saline containing $0.1 \%$ Tween-20 (0.1\% TBST) for analysis by an enzyme-linked immunosorbent assay (ELISA).

Sample preparation for PrP ${ }^{\mathrm{Sc}}$ measurements The precipitation of PK-resistant and PKsensitive $\mathrm{PrP}^{\mathrm{Sc}}$ was performed as described previously (54) with minor modifications. Samples were incubated with peptide-coated magnetic beads (M-280; Invitrogen) for $2 \mathrm{~h}$ at $37^{\circ} \mathrm{C}$ with constant shaking. The beads were washed five times with buffer before denaturation with $0.1 \mathrm{M}$ $\mathrm{NaOH}$ and neutralization with $0.3 \mathrm{M} \mathrm{NaH}_{2} \mathrm{PO}_{4}$. The levels of now disaggregated PrP were then measured by ELISA.

ELISA measurements PrP was measured by a standard ELISA assay using 96-well plates pre-coated with $2.5 \mu \mathrm{g} / \mathrm{ml}$ POM-2 antibody (55). Bound PrP was detected using a biotinylated POM-1 antibody (55) (50 ng/ml), followed by streptavidin-HRP (25 ng/ml) and a 1-Step Ultra TMB-ELISA substrate (Thermo Fisher Scientific) (see Fig. S8D,E). POM-1 was biotinylated using the EZ-Link ${ }^{\mathrm{TM}}$ Sulfo-NHS-LC-Biotinylation Kit (ThermoFisher Scientific). The reaction was stopped by addition of an equal volume of $2 \mathrm{M}$ sulfuric acid, and the plate was read at $450 \mathrm{~nm}$ using an iMark microplate reader (Bio Rad, Hercules CA). RML prion-infected and uninfected control brain samples were included in every experiment. Samples were run in triplicate within each ELISA plate and PrP concentrations interpolated from a standard curve generated using recombinant PrP where possible. Independent ELISAs were performed 3 times. 
Immunoblotting Samples were digested with $20 \mu \mathrm{g} / \mathrm{mL} \mathrm{PK}$, or left undigested, for $30 \mathrm{~min}$ at $37^{\circ} \mathrm{C}$, then mixed with an equal volume of $2 x$ LDS loading buffer (Invitrogen) and heated to $95^{\circ} \mathrm{C}$ prior to electrophoresis through a $10 \%$ Bis-Tris gel (Invitrogen). Samples were transferred to a nitrocellulose membrane by wet blotting. For dot-blotting, $10 \mu \mathrm{l}$ of undigested samples were made up to $50 \mu \mathrm{l}$ with $2 \mathrm{x}$ LDS and transferred to a nitrocellulose membrane using the 96-well Bio-Dot apparatus (BioRad) under vacuum. The membrane was then removed for incubation in the presence of antibodies. Proteins were detected with anti-PrP antibody POM19 followed by an HRP-conjugated anti-mouse IgG antibody (Jackson Immunolabs). Signals were visualized using a chemiluminescent substrate (Supersignal West DuraR, ThermoScientific) and an LAS-4000 imager (Fujifilm).

\section{Standard Scrapie Cell Assay (SSCA) for determination of infectivity CAD5 cells were} grown with standard OFBS Medium (Opti-MEM containing 10\% FBS, 1\% streptomycin and penicillin, 1\%Glutamax; Gibco) in a T150 cell culturing flask. Standard scrapie cell assay was performed according to published protocols (56), with minor modifications. One day prior to infection, 10000 CAD5 and CAD5 KO cells lacking $\operatorname{PrP}^{\mathrm{C}}$ expression were plated with $100 \mu \mathrm{L}$ OFBS in 96-well cell culture plates (TPP) and incubated at $37^{\circ} \mathrm{C}$ with $5 \% \mathrm{CO}_{2}$. On the following day, $100 \mu \mathrm{L}$ of brain homogenate diluted in OFBS mixed with $0.01 \%$ brain homogenate from C57BL/6J-PrnpZH3/ZH3 mice (57) to provide a complex matrix was added to the cells for the infection. To establish a standard curve for infection, a 1:5 serial dilution of RML6 brain homogenate $(20 \% \mathrm{w} / \mathrm{v}$ in $0.32 \mathrm{M}$ sucrose, $109.2 \mathrm{LD} 50$ units per $\mathrm{mL})$ was used with a range from $10^{-3}$ to $6.4 \cdot 10^{-8}$. For each sample, three different dilutions were performed ranging from $10^{-3}$ to $10^{-5}$. To control for residual inoculum, CAD5 KO cells were incubated with RML brain homogenate corresponding to the highest concentration of the standard $(0.01 \%)$. CAD5 cells were incubated with $(0.01 \%)$ non-infectious brain homogenate $(10 \% \mathrm{w} / \mathrm{v}$ in $0.32 \mathrm{M}$ sucrose) to 
control for efficient PK (Roche) digestion and for computing the background of the assay. Three days following infection, cells were split 1:8 into new 96 well plates containing fresh OFBS. After reaching confluence, two additional 1:8 splitting steps were performed, corresponding to days 7 and 10 post infection. On day 14 post infection, ELISPOT membranes (Millipore) were activated by adding $50 \mu \mathrm{L}$ of filtered ethanol/well, washed twice with $160 \mu \mathrm{L}$ PBS and nearly 40000 cells per well transferred onto the membrane and dried with a plate thermomixer (Eppendorf) at $50^{\circ} \mathrm{C}$. After drying, plates were stored at $4^{\circ} \mathrm{C}$ until lysis and digestion. $50 \mu \mathrm{L}$ of $0.5 \mu \mathrm{g} / \mathrm{mL}$ PK in lysis buffer ( $50 \mathrm{mM}$ Tris- $\mathrm{HCl} \mathrm{pH} 8,150 \mathrm{mM} \mathrm{NaCl}, 0.5 \%$ w/v sodium deoxycholate, $0.5 \% \mathrm{w} / \mathrm{v}$ Triton-X-100) was added to each well and incubated for 90 minutes at $37^{\circ} \mathrm{C}$. Following incubation, vacuum was applied to discard the contents and wells were washed twice with $160 \mu \mathrm{L}$ PBS. To stop digestion, $160 \mu \mathrm{L}$ of $2 \mathrm{mM}$ PMSF (Sigma Aldrich) diluted in PBS was applied to the membrane and incubated at room temperature for $10 \mathrm{~min}$. Tris guanidinium thiocyanate was prepared by diluting $3 \mathrm{M}$ guanidinium thiocyanate in $10 \mathrm{mM}$ Tris $\mathrm{HCl}$ $\mathrm{pH} 8$, and added subsequently with a total volume of $160 \mu \mathrm{L} /$ well and incubated for $10 \mathrm{~min}$. Supernatant was discarded into $2 \mathrm{M} \mathrm{NaOH}$ and membrane was washed seven times with each $160 \mu \mathrm{L}$ PBS and blocked $1 \mathrm{~h}$ with $160 \mu \mathrm{L}$ Superblock (Thermo Scientific) prepared in MilliQ. Remaining blocking solution was removed under vacuum and $50 \mu \mathrm{L}$ POM1 antibody (55) was applied at a concentration of 1:5000 diluted in TBST (10 mM Tris $\mathrm{HCl}, \mathrm{pH} 8,150 \mathrm{mM} \mathrm{NaCl}$, $0.1 \%$ (v/v) Tween 20) containing 1\% (w/v) non-fat dry milk for $1 \mathrm{~h}$. Supernatant was discarded and wells were subsequently washed seven times with TBST under vacuum. $50 \mu \mathrm{L}$ of antiIgG1-AP (Southern Biotechnology Associates) was used with a 1:4500 dilution in TBST-1\% (w/v) non-fat dry milk and incubated for $1 \mathrm{~h}$. Discarding of the supernatant and washing was performed in the same way as for the POM1 antibody. $50 \mu \mathrm{L}$ of AP dye (Bio-rad) for the reaction was applied and incubated for 16 minutes. Membrane was washed twice with water, dried and stored at $-20^{\circ} \mathrm{C}$ in dark. Quantifications of the membranes were done using ImageJ 
(open source) with optical density, allowing to distinguish between spots (representing cells that contain PK-resistant PrP) and clear areas.

\section{Measurement of average size of $\mathrm{PK}$ resistant $\mathrm{PrP}^{\mathrm{Sc}}$ aggregates in brain homogenates by}

Immuno-Diffusional Sizing. Sample preparation: The mice brains were washed with ethanol $(2 x, 70 \%)$ and sterile PBS (4x). Then they were homogenised in an eight-fold amount of PBS to prepare a $10 \%$ wt. solution with the Ribolyzer tube (speed 6.5, 4x 35s). After the first homogenisation step the tubes were cooled down on ice and the step was repeated 2 more times. The homogenates were centrifuged ( $700 \mathrm{~g}, 3 \mathrm{~min})$. The supernatant was collected for further use and the pellet was discarded. Before injection into the microfluidic device, the brain homogenate $(\mathrm{BH})$ samples were diluted to $1 \%$ on tris buffer $(50 \mathrm{mM}, \mathrm{pH} 7.4,1 \%$ wt. BSA). PK-digestion: tga20-RML infected 10\% BH were diluted to 2\% in PBS. $10 \mu \mathrm{l}$ of PK (1000 $\mu \mathrm{g} / \mathrm{ml}, \mathrm{C}_{\mathrm{f}}=50 \mu \mathrm{g} / \mathrm{ml}$ ) was added to $190 \mu \mathrm{l}$ of $2 \% \mathrm{BH}$. The mixture was incubated for 2 hours at $37^{\circ} \mathrm{C}$ under continuous shaking at $650 \mathrm{rpm}$. The digestion was stopped by addition of PMSF to a final concentration of $3 \mathrm{mM}$ and incubation for $15 \mathrm{~min}$ with continuous shaking at $750 \mathrm{rpm}$.

Diffusional Sizing in Microfluidic Devices: The samples were analysed with Western Blot. For better results, the sample were injected fresh after PK-digestion directly into the microfluidic device. The samples were loaded in plastic syringes and injected into a microfluidic device with an H-filter geometry (58), using positive flow control with Nemesys syringe pumps. For the non-infected $\operatorname{tga} 20$ sample the flow rates for $\mathrm{BH}$ and buffer were 95 and $105 \mu \mathrm{l} / \mathrm{h}$ respectively, giving a total flow rate of $200 \mu \mathrm{l} / \mathrm{h}$ with a channel width of $200 \mu \mathrm{m}$. For the prion-infected $\operatorname{tga} 20$ the flow rates for $\mathrm{BH}$ and buffer were 38 and $42 \mu \mathrm{l} / \mathrm{h}$ respectively, giving a total flow rate of $80 \mu \mathrm{l} / \mathrm{h}$ with a channel width of $80 \mu \mathrm{m}$. Wider channels were required for the non-infected samples, as the diffusion the small particles detected in those conditions would fully diffuse in a $80 \mu \mathrm{m}$ wide channel (see Supplementary Note 4). 
Immunodetection via TR-FRET ( $\operatorname{Pr}^{C}$ of non-infectious brain homogenate): Concentrations in the diffused and non-diffused fractions were determined using a FRET antibody pair consisting of Eu-POM19 antibody and APC-POM1 antibody, as detailed previously. (43) Each aliquot extracted from the outlets ( $25 \mu \mathrm{l} /$ well, in triplicates) was mixed with the antibody pair, Eu-labelled POM1 and APC-labelled POM19 (5 $\mu \mathrm{l}$ each) in a white 384 Opti-well plate (Perkin Elmer). A standard curve of $\operatorname{PrP}^{C}$ from $9.4 \mathrm{nM}$ to $0.05 \mathrm{nM}$ diluted in Tris buffer (50 mM, pH 7, 0.1\% BSA) was included for every experiment. After shaking (10 min, RT, $300 \mathrm{rpm}$ ), the plate was incubated overnight at $4^{\circ} \mathrm{C}$ before measuring it. The FRET signal was measured using the time-resolved fluorescence mode (emission at $615 \mathrm{~nm}$, second emission at $665 \mathrm{~nm}$ ), with the Envision Platereader (Perkin Elmer).

Immunodetection via ELISA ( $\operatorname{PrP}^{S c}$ after PK-digestion): PK-digested brain homogenate was injected into a microfluidic device. In order to disassemble the fibrils for detection with sandwich ELISA, aliquots of the samples extracted from each of the diffused and non-diffused outlets $(162 \mu \mathrm{l})$ were mixed with $\mathrm{NaOH}(21 \mu \mathrm{l}, 0.5 \mathrm{M})$ and incubated for $10 \mathrm{~min}$ at RT under continuous shaking (700 rpm). $24 \mu \mathrm{l}$ of neutralising buffer, $\mathrm{NaH}_{2} \mathrm{PO}_{4}$ was then added to the mix and incubated for 10 min at RT under continuous shaking (700 rpm). PrP concentrations in the diffused and non-diffused fractions were determined using sandwich ELISA. The selected wells of a transparent 384-well high-binding plate were coated overnight at $4^{\circ} \mathrm{C}$ with $50 \mu \mathrm{l}$ of the capture antibody POM1 $(400 \mathrm{ng} / \mathrm{ml})$ in coating buffer $\left(0.1 \mathrm{M} \mathrm{Na}_{2} \mathrm{CO}_{3} / \mathrm{NaHCO}_{3}\right)$. The plate was aspirated four times in washing buffer (PBS, 1\% Tween) before the addition of 100 $\mu \mathrm{l} /$ well of blocking buffer (5\% TopBlock in PBS-T) and incubated for $2 \mathrm{~h}$ at RT. The plate was washed 4 times four times in washing buffer before addition of the samples. A standard curve of recombinant mPrP was included, from serial dilutions 1:2 starting from $0.217 \mathrm{nM}$ to $53 \mathrm{fM}$. Recombinant and brain samples were loaded into the plate (50 $\mu 1$ per well) and incubated for $1.5 \mathrm{~h}$ at RT. The plate was then washed four times with PBS-T buffer. The detection antibody, 
biotin-labeled POM19 (50 $\mu \mathrm{l}, 400 \mathrm{ng} / \mathrm{ml})$ was added in sample buffer (1\% Top-Block in PBS-T) and incubated for $1 \mathrm{~h}$ at RT. The plate was then washed for times with washing buffer. AvidinHRP (50 $\mu \mathrm{l}, 2.5 \mu \mathrm{g} / \mathrm{ml}$ ) diluted in sample buffer was added and incubated for for $1 \mathrm{~h}$ at RT. The plate was once again washed four times with washing buffer, before adding $50 \mu \mathrm{l} /$ well of stabilised TMB. After 15 min incubation, the reaction was stopped by addition of $\mathrm{H}_{2} \mathrm{SO}_{4}$ $(0.5 \mathrm{M}, 50 \mu \mathrm{l} /$ well $)$ and the absorbance read at a wavelength of $450 \mathrm{~nm}$ using the Envision Platereader (Perkin Elmer).

Calculation of hydrodynamic radius: To convert the measured ratio of diffused to nondiffused signal to a hydrodynamic radius, particle-based simulations were performed (44). The general method was developed and validated in Arosio et al. (59) and we here performed additional validation using the same device design and a FRET detection on well-characterised insulin aggregates (see Extended Data Fig.S7for simulation results and validation).

Fitting of $\operatorname{PrP}^{\mathrm{Sc}}$ concentrations to obtain replication rates. The fits of the fits of the data to a logistic function, equation (1), was performed by simple least squares assuming homoscedastic noise. The noise in the measurement itself (ELISA) is expected to be largely independent of the signal strength. The slight increase in noise at later times thus likely reflects the animalto-animal variation. To minimise the complexity of the model and the danger of over-fitting we do not attempt to model this variation explicitly and instead assume homoscedastic noise. Allowing for heteroscedasticity is unlikely to change the results, given that our conclusions are robust even with respect to fitting the data with a different model (see Supplementary Note 2).

\section{Acknowledgments}

Thanks to Petra Schwarz for technical help. We acknowledge funding from Sidney Sussex College Cambridge (GM), the Mexican National Council of Science and Technology (ICM) 
and Cambridge Trust (ICM), the Synapsis foundation (SS), the Amyloidosis Foundation (MN),

The datasets generated and/or analysed during the current study are available from the corresponding authors on reasonable request. 